\title{
Laparoscopic Surgery for Gallbladder Cancer: An Expert Consensus Statement
}

\author{
Ho-Seong Han ${ }^{\mathrm{a}}$ Yoo-Seok Yoon ${ }^{\mathrm{a}}$ Anil K. Agarwal ${ }^{\mathrm{b}}$ Giulio Bellic \\ Osamu Itano $^{d}$ Andrew A. Gumbs ${ }^{e}$ Dong Sup Yoon ${ }^{f}$ Chang Moo Kang ${ }^{f}$ \\ Seung Eun Lee ${ }^{g}$ Toshifumi Wakai ${ }^{\text {h }}$ Roberto I. Troisi ${ }^{i}$ \\ ${ }^{a}$ Seoul National University Bundang Hospital, Seongnam, South Korea; ${ }^{b}$ GB Pant Hospital, Delhi, India; ${ }^{\mathrm{C} S}$.M. \\ Loreto Nuovo Hospital, Naples, Italy; ${ }^{\mathrm{d}}$ International University of Health and Welfare School of Medicine, Chiba,

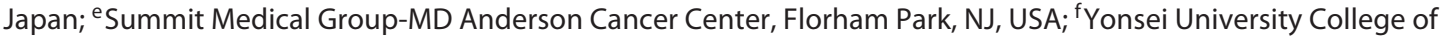 \\ Medicine, Seoul, South Korea; ${ }^{9}$ Chung-Ang University College of Medicine, Seoul, South Korea; ${ }^{h}$ Niigata University \\ Graduate School of Medical and Dental Sciences, Niigata, Japan; 'Ghent University Hospital and Medical School, \\ Ghent, Belgium
}

\section{Keywords \\ Laparoscopic surgery · Gallbladder cancer · Consensus · Expert}

\begin{abstract}
Background: Despite the increasing number of reports on the favorable outcomes of laparoscopic surgery for gallbladder cancer $(\mathrm{GBC})$, there is no consensus regarding this surgical procedure. Objective: The study aimed to develop a consensus statement on the application of laparoscopic surgery for GBC based on expert opinions. Methods: A consensus meeting among experts was held on September 10, 2016, in Seoul, Korea. Results: Early concerns regarding port site/ peritoneal metastasis after laparoscopic surgery have been abated by improved preoperative recognition of GBC and careful manipulation to avoid bile spillage. There is no evidence that laparoscopic surgery is associated with decreased survival compared with open surgery in patients with earlystage $G B C$ if definitive resection during/after laparoscopic cholecystectomy is performed. Although experience with laparoscopic extended cholecystectomy for GBC has been limited to a few experts, the postoperative and survival outcomes were similar between laparoscopic and open surger-
\end{abstract}

ies. Laparoscopic reoperation for postoperatively diagnosed GBC is technically challenging, but its feasibility has been demonstrated by a few experts. Conclusions: Laparoscopic surgery for $\mathrm{GBC}$ is still in the early phase of the adoption curve, and more evidence is required to assess this procedure.

(c) 2018 S. Karger AG, Basel

\section{Introduction}

The application of laparoscopic approaches to treat gastrointestinal malignancies is increasing. Initial concerns about tumor cell dissemination and the inadequacies of radical surgery related to laparoscopic surgery have been resolved by high-quality prospective studies on laparoscopic surgery in colonic and gastric cancer patients $[1,2]$. Currently, this procedure is widely accepted to treat typical gastrointestinal malignancies. However,

This meeting was held during the 26th World Congress of the International Association of Surgeons, Gastroenterologists and Oncologists (IASGO) in Seoul in 2016.

\section{KARGER}

(c) 2018 S. Karger AG, Basel

E-Mail karger@karger.com

www.karger.com/dsu
Prof. Yoo-Seok Yoon

Department of Surgery

Seoul National University Bundang Hospital

166 Gumi-ro, Bundang-gu, Seongnam-si, Gyeonggi-do 463-707 (South Korea)

E-Mail yoonys@snubh.org 
laparoscopic surgery for gallbladder cancer (GBC) is still in the early phase of the adoption curve. A laparoscopic approach for GBC is still controversial among hepatobiliary/pancreatic surgeons. Although this procedure has been contraindicated in patients with GBC for some time, many recent reports have shown that laparoscopic surgery does not adversely affect the perioperative and survival outcomes of patients with GBC [3-9]. One recent report showed that laparoscopic extended cholecystectomy for GBC achieved an outcome comparable with that of open surgery over long-term follow-up [10].

With this background, experts convened at a consensus meeting with the purpose of defining the current state of laparoscopic surgery for GBC and obtaining expert suggestions on the application of this procedure. Here, we report an expert panel statement for laparoscopic surgery for $\mathrm{GBC}$ compiled during the meeting.

\section{Methods}

An expert consensus meeting was held on September 10, 2016, during the 26th World Congress of the International Association of Surgeons, Gastroenterologists and Oncologists. International experts in GBC laparoscopic surgery were invited to participate in the meeting and to present specific issues regarding laparoscopic surgery for GBC, including oncologic concerns, selection criteria, surgical techniques, and future aspects of this procedure. Presentations were followed by panel discussions and open discussions with the audience. After the meeting, a first draft including summaries of the presentations and discussions was circulated to the panels, discussed, and edited. The present document, including expert consensus statements, was formulated and agreed on by all attending experts in this field.

\section{Results}

\section{Concerns Regarding Laparoscopic Surgery for GBC}

Laparoscopic surgery has been contraindicated for the treatment of GBC for the following reasons: (1) risk of port site recurrence or peritoneal metastasis due to bile leak associated with GB injury and (2) concerns regarding the oncologic adequacy and safety of laparoscopic radical surgery.

Port site recurrence or peritoneal dissemination was previously estimated to occur at a relatively high rate (10$18 \%$ ) and to be associated with a poor prognosis in patients with GBC after laparoscopic cholecystectomy [11, 12]. However, these findings were based largely on older studies, in which bile leakage occurred mostly in patients with unsuspected GBC. With improved preoperative recognition of GBC and implementation of a plastic bag to remove the resected GB, no significant differences in the occurrence of port site/wound or peritoneal metastases or in survival outcomes have been observed between laparoscopic and open surgeries. Nonetheless, bile spillage, if it occurs, remains a risk factor for tumor recurrence and reduced survival. Therefore, when GBC is suspected, careful manipulation is required to avoid bile spillage.

If GBC extends beyond the muscular layer, cancer cells may occur on the hepatic side of the GB, because dissection on the hepatic side is performed in the subserosal layer during standard cholecystectomy. Although an accurate preoperative/intraoperative diagnosis regarding the depth of mural invasion is very important to avoid this problem, the currently available diagnostic tools have limitations. Therefore, cholecystectomy inclusive of the cystic plate or a thin layer of liver parenchyma, even for early-stage GBC, and sometimes en-bloc liver resection of the GB bed, can serve as preventive measures to reduce the risks of microscopic residual cancer and GB perforation. With accumulation of experience, laparoscopic lymph node (LN) dissection, which is widely performed to treat colonic and gastric cancers, has become a safe and adequate procedure in oncologic contexts.

\section{Consensus Statements}

- Early concerns regarding port site/peritoneal metastasis after laparoscopic surgery for GBC have subsided with improved preoperative recognition of GBC and preventive measures such as using a plastic bag for removal of GB specimens.

- When GBC is suspected, perforation of the GB should be prevented by cautious and meticulous techniques.

- During cholecystectomy for early-stage GBC, cholecystectomy inclusive of the cystic plate or a thin layer of liver parenchyma is recommended to reduce the risks of GB perforation and residual cancer on the hepatic side.

\section{Application of Laparoscopic Surgery for GBC}

Open surgery has been recommended even for suspected early-stage GBC because of the abovementioned concerns regarding laparoscopic surgery. However, with the increasing incidental detection of GBC resulting from widespread application of laparoscopic cholecystectomy, many reports have shown that laparoscopic surgery does not adversely influence the prognosis of patients with early-stage GBC. When definitive oncologic resection is performed for GBC detected incidentally during surgery or that diagnosed postoperatively, survival was not adversely affected by laparoscopic surgery $[13,14]$. 
Based on these results, laparoscopic cholecystectomy may be a feasible option for suspected GBC unless there is preoperative evidence of liver invasion. Cholecystectomy should be performed according to the abovementioned principles. However, the outcome of patients with GBC after laparoscopic surgery for acute cholecystitis is concerning [15-17]. Therefore, laparoscopic surgery may be better indicated for GBC that is not associated with acute cholecystitis.

\section{Consensus Statements}

- There is no evidence that laparoscopic surgery is associated with decreased survival compared with open surgery in patients with a suspicion of early-stage GBC if definite resection during/after laparoscopic cholecystectomy is performed.

- Laparoscopic surgery is not yet indicated for GBC associated with acute cholecystitis.

\section{Laparoscopic Extended Cholecystectomy for GBC Patient Selection}

Patients with GBC have various clinical presentations requiring different surgical extents, from a small polypoid mass to a large mass invading the GB bed, hilum of the liver, or other adjacent organs. Among these, the appropriate patient group for GBC laparoscopic surgery should be carefully selected for oncologic safety during the initial period of adoption. In terms of depth of mural invasion, the indications for laparoscopic extended cholecystectomy (defined as cholecystectomy + dissection of regional LNs \pm liver resection inclusive of the GB bed) in recent reports have been mostly stage T1 and T2 GBC; reports on this procedure for stage T3 $\mathrm{GBC}$ are limited.

Although the T stage (T1b or T2) at which extended cholecystectomy should be required is still controversial, decision-making on this should follow the same criteria for laparoscopic surgery as for open surgery. Accurate preoperative staging in terms of invasion depth is crucial, because this is used to determine the surgical approach and prognosis. Although conventional abdominal ultrasonography (US) and computed tomography are widely used for preoperative staging, these modalities are limited in their ability to predict the depth of invasion compared with endoscopic, high-resolution, or laparoscopic US, which have higher resolution and thus are better able to delineate the multiple layers of the GB wall $[18,19]$. A recent intention-totreat analysis of intended laparoscopic surgery showed favorable clinical and survival outcomes in patients with

Laparoscopic Surgery for Gallbladder Cancer
GBC who were highly selected by multimodality imaging studies, including endoscopic and laparoscopic US $[3,10]$.

\section{Technique}

The technical concerns regarding laparoscopic radical surgery for $\mathrm{GBC}$ are adequate $\mathrm{LN}$ dissection, margin-free liver resection, and bile duct resection/reconstruction when needed. Despite limited experiences by experts, laparoscopic extended cholecystectomy has been shown to be safe and feasible in selected GBC patients, with similar results to those of open surgery $[3,5,7-10,18,20-26]$. According to published reports, the most commonly performed procedures are wedge liver resection and LN dissection. Laparoscopic bile duct resection may be performed in cases with positive cystic duct margins or involvement of the bile duct by tumor. Laparoscopic IVb/V segmentectomy has been performed in some centers $[5,9,25]$.

\section{LN Dissection}

Laparoscopic LN dissection is now widely performed for colonic and gastric cancers, and its oncologic efficacy has been well established. Experience with these gastrointestinal cancers has provided a foundation for applying this procedure for GBC. There is no consensus on the optimal extent of LN dissection even for open extended cholecystectomy. The extent of LN dissection for GBC in all published reports includes removal of LNs around the hepatoduodenal ligament. Some centers additionally dissect LNs in the posterior superior pancreaticoduodenal area and along the common hepatic artery because of frequent LN metastasis in this area and improved patient survival if removed completely [27-29]. Recent reports have shown that LN dissection can be performed to the same extent in laparoscopic surgery as in the open procedure in selected GBC patients with few intraoperative and postoperative complications $[3,5,7-10]$. The number of retrieved LNs is comparable between laparoscopic and open surgeries.

The role of more extensive dissection of aorto-caval, celiac, superior mesenteric artery nodes seems limited, because no survival benefit is evident. However, aorto-caval LN sampling at the initiation of surgery is advocated in some centers because of frequent involvement of these LNs. This concept applies to laparoscopic surgery as well.

\section{Liver Resection}

The aims of liver resection in patients with GBC are to achieve a negative resection margin on the hepatic side and to minimize the likelihood of recurrence by removal 
of microscopic metastases in the liver. There is no consensus on the optimal extent of liver resection between wedge resection and segment $\mathrm{IVb} / \mathrm{V}$ resection even in open surgery. Theoretical advantages of segment IVb/V resection over wedge resection have not been demonstrated clinically. No significant difference in survival rate between the 2 procedures has been reported. Recent reports on laparoscopic extended cholecystectomy for GBC have demonstrated the technical feasibility and safety of laparoscopic wedge resection, and the feasibility of laparoscopic $\mathrm{IVb} / \mathrm{V}$ resection for $\mathrm{GBC}$ has also been reported $[5,9,25]$.

\section{Bile Duct Resection}

Routine resection of the bile duct for $\mathrm{LN}$ dissection is not recommended, because it increases morbidity with no evidence of improving survival [30-33]. Indications for this procedure include a positive cystic duct margin after cholecystectomy to obtain a negative margin, direct tumor invasion of the bile duct, and inflammation or scarring around the hepatoduodenal ligament compromising complete LN dissection. The feasibility of laparoscopic bile duct excision for GBC has been reported in the literature [23]. Considering the increasing reports on laparoscopic surgery for choledochal cysts and laparoscopic pancreatoduodenectomy, the necessity of bile duct resection is not a contraindication for GBC laparoscopic surgery.

\section{Survival Outcomes}

The reported survival outcomes of patients with GBC after laparoscopic extended cholecystectomy are encouraging. The recurrence and survival rates were found to be similar between laparoscopic and open surgery groups in comparative studies $[5,7]$. Furthermore, the favorable long-term outcome of laparoscopic extended cholecystectomy was shown in a recent study, in which the survival rate of patients at 5 years postoperatively was over $90 \%$ [10]. However, considering the small numbers of included patients and the retrospective nature of those previous studies, further large-scale comparative studies are needed to confirm the oncologic safety of laparoscopic extended cholecystectomy for GBC.

\section{Consensus Statement}

- Experience with laparoscopic extended cholecystectomy for GBC has been limited to a few expert centers.

- The reports on laparoscopic extended cholecystectomy have shown acceptable postoperative outcomes compared with those of open surgery.
- For safe selection of indicated patients, accurate preoperative staging in terms of depth of invasion is important. Endoscopic or laparoscopic US may be more useful as a complementary procedure compared with conventional US and computed tomography for this purpose.

- The most common type of liver resection reported is wedge resection of the GB bed. Laparoscopic IVb/V segmentectomy is performed in some centers. As there are limited reports on the outcomes after laparoscopic surgery for GBC with liver invasion, more data are needed.

- Laparoscopic LN dissection for early GBC was shown to be a safe and adequate procedure from an oncologic point of view.

- Bile duct resection is not a contraindication for laparoscopic surgery for GBC.

- Although the evidence is limited, the survival outcomes of highly selected patients are similar between laparoscopic and open surgeries.

\section{Laparoscopic Reoperation for Postoperatively}

\section{Diagnosed GBC}

The reported incidence of postoperatively diagnosed GBC after cholecystectomy is $0.19-3.3 \%$ [34]. Widespread adoption of laparoscopic cholecystectomy has resulted in an apparent increase in this rate. Radical reoperation after cholecystectomy is decided based on the postoperative pathology and is usually performed via open surgery. This procedure is considered technically challenging mainly because of inflammatory adhesions or fibrosis around the hepatoduodenal ligament and GB bed. There are a few recent reports on the technical feasibility of laparoscopic revisional surgery $[5,8,21,22,26$, 35]. Unlike initial laparoscopic surgery for GBC, laparoscopic reoperation is not associated with the risk of tumor seeding related to bile spillage, because cholecystectomy has already been performed. Despite very limited experience by experts, a laparoscopic approach for postoperatively diagnosed GBC seems to be feasible, with satisfactory clinical outcomes in terms of perioperative and midterm oncologic results.

In patients with postoperatively diagnosed GBC, port site metastasis has been a concern during the early stage of laparoscopy. Although its incidence is rare, port site metastasis is associated with poor survival once it develops. However, routine port site resection is not recommended during definitive surgical treatment, because it has not been associated with improved survival or reduced recurrence [36].
Han/Yoon/Agarwal/Belli/Itano/Gumbs/ Yoon/Kang/Lee/Wakai/Troisi 


\section{Consensus Statement}

- Laparoscopic reoperation for postoperatively diagnosed GBC is technically challenging, but its feasibility has been demonstrated by some expert teams.

- During this procedure, routine excision of the port site is not recommended, because there is no evidence of improved survival.

\section{Conclusion}

With preoperative recognition of $\mathrm{GBC}$ and careful manipulation to avoid bile spillage, laparoscopic surgery does not worsen the prognosis of patients with early- stage GB if definitive resection during/after laparoscopic cholecystectomy is conducted. Although experience with laparoscopic extended cholecystectomy for GBC has been limited to a few experts, the postoperative and survival outcomes of highly selected patients were favorable. However, laparoscopic surgery for GBC is still in the early phase of the adoption curve, and more evidence is required before this procedure can be widely accepted.

\section{Disclosure Statement}

The authors have no conflicts of interest to declare.

\section{References}

1 Lacy AM, Garcia-Valdecasas JC, Delgado S, Castells A, Taura P, Pique JM, Visa J: Laparoscopy-assisted colectomy versus open colectomy for treatment of non-metastatic colon cancer: a randomised trial. Lancet 2002;359: 2224-2229.

-2 Kim YW, Yoon HM, Yun YH, Nam BH, Eom BW, Baik YH, Lee SE, Lee Y, Kim YA, Park JY, Ryu KW: Long-term outcomes of laparoscopy-assisted distal gastrectomy for early gastric cancer: result of a randomized controlled trial (COACT 0301). Surg Endosc 2013;27: 4267-4276.

- 3 Cho JY, Han HS, Yoon YS, Ahn KS, Kim YH, Lee KH: Laparoscopic approach for suspected early-stage gallbladder carcinoma. Arch Surg 2010;145:128-133.

4 Lee SE, Jang JY, Lim CS, Kang MJ, Kim SW: Systematic review on the surgical treatment for T1 gallbladder cancer. World J Gastroenterol 2011;17:174-180.

5 Agarwal AK, Javed A, Kalayarasan R, Sakhuja P: Minimally invasive versus the conventional open surgical approach of a radical cholecystectomy for gallbladder cancer: a retrospective comparative study. HPB (Oxford) 2015; 17:536-541.

6 Gumbs AA, Jarufe N, Gayet B: Minimally invasive approaches to extrapancreatic cholangiocarcinoma. Surg Endosc 2013;27:406414.

7 Itano O, Oshima G, Minagawa T, Shinoda M, Kitago M, Abe Y, Hibi T, Yagi H, Ikoma N, Aiko S, Kawaida M, Masugi Y, Kameyama K, Sakamoto M, Kitagawa Y: Novel strategy for laparoscopic treatment of pT2 gallbladder carcinoma. Surg Endosc 2015;29:3600-3607.

8 Shirobe T, Maruyama S: Laparoscopic radical cholecystectomy with lymph node dissection for gallbladder carcinoma. Surg Endosc 2015 29:2244-2250.

9 Palanisamy S, Patel N, Sabnis S, Palanisamy N, Vijay A, Palanivelu P, Parthasarthi R,
Chinnusamy P: Laparoscopic radical cholecystectomy for suspected early gall bladder carcinoma: thinking beyond convention. Surg Endosc 2016;30:2442-2448.

-10 Yoon YS, Han HS, Cho JY, Choi Y, Lee W, Jang JY, Choi H: Is laparoscopy contraindicated for gallbladder cancer? A 10-year prospective cohort study. J Am Coll Surg 2015; 221:847-853.

11 Reddy YP, Sheridan WG: Port-site metastasis following laparoscopic cholecystectomy: a review of the literature and a case report. Eur J Surg Oncol 2000;26:95-98.

12 Berger-Richardson D, Chesney TR, Englesakis M, Govindarajan A, Cleary SP, Swallow CJ: Trends in port-site metastasis after laparoscopic resection of incidental gallbladder cancer: a systematic review. Surgery 2017;161: 618-627.

13 Ouchi K, Mikuni J, Kakugawa Y; Organizing Committee The 30th Annual Congress of the Japanese Society of Biliary Surgery: Laparoscopic cholecystectomy for gallbladder carcinoma: results of a Japanese survey of $498 \mathrm{pa}$ tients. J Hepatobiliary Pancreat Surg 2002;9: 256-260.

14 Whalen GF, Bird I, Tanski W, Russell JC, Clive J: Laparoscopic cholecystectomy does not demonstrably decrease survival of patients with serendipitously treated gallbladder cancer. J Am Coll Surg 2001;192:189195.

15 Clemente G, Nuzzo G, De Rose AM, Giovannini I, La Torre G, Ardito F, Giuliante F: Unexpected gallbladder cancer after laparoscopic cholecystectomy for acute cholecystitis: a worrisome picture. J Gastrointest Surg 2012; 16:1462-1468.

16 Kim JH, Kim WH, Kim JH, Yoo BM, Kim MW: Unsuspected gallbladder cancer diagnosed after laparoscopic cholecystectomy: focus on acute cholecystitis. World J Surg 2010; 34:114-120.
17 Han HS, Cho JY, Yoon YS, Ahn KS, Kim H: Preoperative inflammation is a prognostic factor for gallbladder carcinoma. Br J Surg 2011;98:111-116.

18 Jang JY, Kim SW, Lee SE, Hwang DW, Kim EJ, Lee JY, Kim SJ, Ryu JK, Kim YT: Differential diagnostic and staging accuracies of high resolution ultrasonography, endoscopic ultrasonography, and multidetector computed tomography for gallbladder polypoid lesions and gallbladder cancer. Ann Surg 2009;250: 943-949.

19 Fujita N, Noda Y, Kobayashi G, Kimura K, Yago A: Diagnosis of the depth of invasion of gallbladder carcinoma by EUS. Gastrointest Endosc 1999;50:659-663.

20 Cho A, Yamamoto H, Nagata M, Takiguchi N, Shimada H, Kainuma O, Souda H, Gunji H, Miyazaki A, Ikeda A, Matsumoto I: Total laparoscopic resection of the gallbladder together with the gallbladder bed. J Hepatobiliary Pancreat Surg 2008;15:585-588.

-21 de Aretxabala X, Leon J, Hepp J, Maluenda F, Roa I: Gallbladder cancer: role of laparoscopy in the management of potentially resectable tumors. Surg Endosc 2010;24:2192-2196.

22 Gumbs AA, Hoffman JP: Laparoscopic completion radical cholecystectomy for T2 gallbladder cancer. Surg Endosc 2010;24:32213223.

23 Gumbs AA, Hoffman JP: Laparoscopic radical cholecystectomy and Roux-en-Y choledochojejunostomy for gallbladder cancer. Surg Endosc 2010;24:1766-1768.

24 Gumbs AA, Milone L, Geha R, Delacroix J, Chabot JA: Laparoscopic radical cholecystectomy. J Laparoendosc Adv Surg Tech A 2009; 19:519-520.

25 Machado MA, Makdissi FF, Surjan RC: Totally laparoscopic hepatic bisegmentectomy $(s 4 b+s 5)$ and hilar lymphadenectomy for incidental gallbladder cancer. Ann Surg Oncol 2015;22(suppl 3):336-339. 
-26 Yamashita S, Loyer E, Chun YS, Javle M, Lee JE, Vauthey JN, Conrad C: Laparoscopic management of gallbladder cancer: a stepwise approach. Ann Surg Oncol 2016;23(supple 5):892-893.

27 Chijiiwa K, Noshiro H, Nakano K, Okido M, Sugitani A, Yamaguchi K, Tanaka M: Role of surgery for gallbladder carcinoma with special reference to lymph node metastasis and stage using western and Japanese classification systems. World J Surg 2000;24:12711276.

28 Lee SE, Jang JY, Lim CS, Kang MJ, Kim SW Systematic review on the surgical treatment for T1 gallbladder cancer. World J Gastroenterol 2011;17:174-180.

-29 Miyazaki M, Yoshitomi H, Miyakawa S, Uesaka K, Unno M, Endo I, Ota T, Ohtsuka M, Kinoshita H, Shimada K, Shimizu H, Tabata M, Chijiiwa K, Nagino M, Hirano S, Wakai T, Wada K, Isayama H, Okusaka T, Tsuyu- guchi T, Fujita N, Furuse J, Yamao K, Murakami K, Yamazaki H, Kijima H, Nakanuma Y, Yoshida M, Takayashiki T, Takada T: Clinical practice guidelines for the management of biliary tract cancers 2015: the 2nd English edition. J Hepatobiliary Pancreat Sci 2015;22:249-273.

30 D’Angelica M, Dalal KM, DeMatteo RP, Fong Y, Blumgart LH, Jarnagin WR: Analysis of the extent of resection for adenocarcinoma of the gallbladder. Ann Surg Oncol 2009;16:806816.

31 Fuks D, Regimbeau JM, Le Treut YP, Bachellier P, Raventos A, Pruvot FR, Chiche L, Farges O: Incidental gallbladder cancer by the AFC-GBC-2009 Study Group. World J Surg 2011;35:1887-1897.

32 Nigri G, Berardi G, Mattana C, Mangogna L, Petrucciani N, Sagnotta A, Aurello P, D'Angelo F, Ramacciato G: Routine extra-hepatic bile duct resection in gallbladder cancer patients without bile duct infiltration: a systematic review. Surgeon 2016;14:337-344.

33 Shukla PJ, Barreto SG: Systematic review: should routine resection of the extra-hepatic bile duct be performed in gallbladder cancer? Saudi J Gastroenterol 2010;16:161-167.

- 34 Sujata J, S R, Sabina K, Mj H, Jairajpuri ZS: Incidental gall bladder carcinoma in laparoscopic cholecystectomy: a report of 6 cases and a review of the literature. J Clin Diagn Res 2013;7:85-88.

35 Belli G, Cioffi L, D’Agostino A, Limongelli P, Belli A, Russo G, Fantini C: Revision surgery for incidentally detected early gallbladder cancer in laparoscopic era. J Laparoendosc Adv Surg Tech A 2011;21:531-534.

36 Maker AV, Butte JM, Oxenberg J, Kuk D, Gonen M, Fong Y, Dematteo RP, D’Angelica MI, Allen PJ, Jarnagin WR: Is port site resection necessary in the surgical management of gallbladder cancer? Ann Surg Oncol 2012;19:409-417. 